\title{
Um Modelo Ubíquo de Detecção de Riscos de Alergia Baseado em Ciência de Situação
}

\author{
Nelson Manoel de Moura Quevedo; Cristiano André da Costa; \\ Rodrigo da Rosa Righi; Sandro José Rigo
}

\author{
Programa de Pós- Graduação em Computação Aplicada (PIPCA) \\ Universidade do Vale do Rio dos Sinos (Unisinos) - São Leopoldo, RS - Brasil. \\ nquevedo@hotmail.com; \{cac, rrrighi, rigo $\}$ dunisinos.br
}

\begin{abstract}
Advances in ubiquitous computing are enabling the emergence of opportunities in many areas, among them is the health area. In this area occur many applications that enable ubiquitous care, called U-Healthcare applications. A detailed survey about existing and proposed models has shown that none of these applications meets people who suffer from food allergy. Thus, this paper proposes to present a ubiquitous model of allergy detection based on situation awareness, called Allergy Detector. This model focuses in food allergy, in particular the eight major allergens (peanut, milk, egg, wheat, soy, fish, crustacean and tree nuts) and their derivate, who causes about $90 \%$ of all food allergies. In order to assess the model, a study of case was designed and applied in a prototype that demonstrated the proposal viability.
\end{abstract}

Resumo. Avanços em computação ubíqua estão permitindo o surgimento de oportunidades em diversas áreas, entre elas está a área da saúde. Nessa área surgem diversas aplicações que possibilitam cuidados ubíquos, chamados de aplicações de U-Healthcare. Um estudo detalhado dos modelos e propostas existentes, mostrou que nenhuma dessas aplicações atende pessoas que sofrem de alergia alimentar. Assim, a proposta desse trabalho é apresentar um modelo ubíquo de detecção de riscos de alergia baseado em ciência de situação, chamado de Allergy Detector. O modelo é focado em alergia alimentar, em particular aos oito principais alergênicos (amendoim, leite, ovo, trigo, soja, peixe, crustáceos e amendoas oriundas de árvores) e seus derivados, responsáveis por $90 \%$ de todas as reações de alergias alimentares. Para avaliar o modelo foi desenvolvido um cenário de teste e aplicado em um protótipo que demonstrou a viabilidade da proposta.

\section{Introdução}

A computação ubíqua está permitindo o surgimento de oportunidades em diversas áreas como saúde, educação, jogos, comércio, entretenimento e transportes (Feltes e Barbosa, 2014). Especificamente na saúde, a área de Cuidados Ubíquos ou "U-Healthcare" é muito promissora nos dias de hoje, onde a ubiquidade é a resposta para o fornecimento de serviços médicos em qualquer lugar e a qualquer momento. $\mathrm{O}$ objetivo de $\mathrm{U}$ Healthcare é fornecer serviço de saúde conveniente, tanto para profissionais da saúde como para usuários, de maneira a tornar fácil o diagnóstico do estado de saúde do usuário (Gelogo e Kim, 2013). 
Existem modelos que fornecem cuidados ubíquos tais como planejamento alimentar (Antoniou e Nanou, 2003), recomendação evitando alimentos calóricos (Johnson, Vergara e Doll, 2014), sugestão de restaurante (Daraghmi e Yuan, 2013), diário dos alimentos ingeridos (Henricksen e Viller, 2012) e seleção de menus com restrições para uma dieta segura (Iizuka e Okawada, 2012). Porém nenhum dos modelos estudados provê suporte de maneira ubíqua a usuários que sofrem de alergia alimentar.

As reações alimentares de causas alérgicas acometem 6-8\% das crianças com menos de 3 anos de idade e 2-3\% dos adultos (Associação Brasileira de Alergia e Imunologia, ASBAI). Ademais, a única terapia comprovadamente eficaz contra alergia alimentar é a exclusão dietética do alergênico ao usuário (Silva et al., 2008).

Assim, o objetivo principal do trabalho é a criação de um modelo para detectar riscos a usuários que sofrem de alergia alimentar aos oito principais alergênicos (soja, ovo, leite, trigo, peixe, crustáceo, amêndoas oriundas de árvores e amendoim) e seus derivados, responsáveis por mais de $90 \%$ dos casos de alergias alimentares (Pereira et al., 2008). O modelo proposto, denominado Allergy Detector, considera quais são as proteínas contidas nos oito principais alergênicos, obtidas a partir da base de dados alergênicos internacional. A principal contribuição científica do modelo consiste em empregar a ciência de situação, definida como a percepção dos elementos de um ambiente dentro de um determinado período de tempo e de espaço, a compreensão dos significados desses elementos e da projeção dos seus estados em um futuro próximo (Endsley, 1999), para o propósito específico de detectar riscos a usuários na área de alergia alimentar. Os elementos no Allergy Detector são as informações do nome e dos pratos/ingredientes do restaurante (contexto de localização), as informações de alergias do usuário (contexto de perfil) e as informações dos oito principais alergênicos e suas proteínas. O processamento trata das inferências permitidas pela ontologia no domínio de alergia alimentar e das informações de contextos de perfil e localização. E finalmente a projeção futura é a inferência do risco de ingestão dos oito principais alergênicos ou de seus derivados. As características ubíquas do modelo estão na mobilidade permitida, do mínimo de interação necessária para detecção do risco e da transparência das ferramentas envolvidas no modelo como um todo.

$\mathrm{O}$ artigo possui seis seções. A seção 2 apresenta alguns conceitos fundamentais para o trabalho. O modelo proposto é descrito na seção 3. A seção 4 apresenta a avaliação e discussão dos resultados. Os trabalhos relacionados são apresentados na seção 5. Por fim, a seção 6 apresenta a conclusão e projeta trabalhos futuros.

\section{Alergia Alimentar e Ciência de Situação}

Reações alimentares são extremamente comuns e geralmente são atribuídas a alergias, podendo ser classificadas como imune mediada, ou não-imune mediada, chamadas de intolerância alimentar. Reações imune mediada podem ser subdivididas em reações mediadas por imunoglobulina E (IgE) ou não (não-IgE) (Guandalini e Newland, 2011).

As reações mediadas por IgE decorrem da sensibilização a alergênicos alimentares com formação de anticorpos específicos da classe IgE e os exemplos de manifestações mais comuns são: reações cutâneas, gastrointestinais, respiratória e reações sistêmicas (anafilaxia com hipertensão e choque) (Silva et al., 2008). 
A principal base de dados de alergênicos mundial é a do International Union of Immunological Societies (IUIS), disponível no site do IUIS ${ }^{1}$, reconhecida como oficial pela OMS. O IUIS é responsável pela manutenção dessa base de dados, pela análise e aprovação de novos alergênicos, e pela nomenclatura das proteínas alergênicas. A regra de nomenclatura consiste da concatenação das três primeiras letras do nome do gênero da fonte alergênica, com a primeira letra da espécie da fonte alergênica e com um número decimal que identifica a ordem em que proteína dessa fonte alergênica foi identificada. Por exemplo, o alergênico do leite de vaca referente à proteína betalactoglobulina foi registrado pelo IUIS com o nome "Bos d 5", que levou em consideração o nome do gênero da vaca "Bos Domesticus" e da espécie em inglês "Domestic Cattle" além do número 5 (Arruda, Moreno e Ferreira, 2013).

A partir das informações existentes na base alergênica é possível o emprego da ciência de situação para identificar possíveis intolerâncias alimentares. Para possibitar a detecção de riscos, o modelo utiliza ciência de situação, baseado no modelo de Endsley que emprega três níveis (Endsley, 1999). Nessa abordagem, os níveis 1 e 2 coletam informações e as processam através de inferências agregando valores. No nível 3, é inferida a classificação do risco do local onde o usuário se encontra, bem como identificados pratos que contém ingredientes alergênicos aos usuário e as proteínas alergênicas contidas nos pratos. Para possibilitar as inferências dos níveis 2 e 3, é necessário a criação de uma ontologia (Feltes e Barbosa, 2014).

\section{Modelo Allergy Detector}

O Allergy Detector foi desenvolvido para detectar riscos a usuários que sofrem de alergia alimentar causadas pelas proteínas dos oito principais alergênicos ou de seus derivados, utilizando o modelo de Endsley como referência.

A Figura 1 apresenta a arquitetura do modelo proposto, que apresenta uma arquitetura baseada em serviço. No lado cliente ficam os módulos que irão executar no dispositivo móvel e que serão responsáveis pela interação com o usuário bem como aquisição de informações de contexto de localização. O cliente vai interagir com um serviço que armazena os módulos responsáveis pelo processamento das informações, realização de inferências e pelo gerenciamento da ciência de situação.

Os serviços executados no lado cliente são: (i) Cadastro de Restaurante: informação da URL onde o restaurante disponibiliza as informações de pratos e ingredientes; (ii) Cadastro de Perfil: informar novas alergias ou dados de autenticação; (iii) Notificador: notificar o usuário através de sinaleira se o local é seguro, incerto ou inseguro se referindo ao risco do usuário ingerir alimentos que causem alergia alimentar ao mesmo e apresentar os pratos com alergênicos e suas proteínas e (iv) Checkin: coletar as informações de contexto de localização, como localização do usuário e nome do restaurante e enviá-las para o servidor de aplicação.

O serviço Allergy Detector possui dois componentes principais: o Serviço de Ontologia e o Servidor de Aplicação O módulo raciocinador do Serviço de Ontologia é o que obtém da ontologia as informações de pratos e ingredientes do restaurante informado pelo módulo checkin. Esse módulo infere se os ingredientes pertencem aos

\footnotetext{
${ }^{1}$ www.allergen.org
} 
oitos principais alergênicos ou a seus derivados, além de identificar na ontologia as proteínas associadas. Uma vez isso feito, o racionador define se os ingredientes dos pratos são alergênicos ao perfil do usuário e constroi uma tabela com os pratos, ingredientes alergênicos ao usuário ou derivados desses, nome da proteína (IUIS), relacionada a cada ingrediente alergênico.

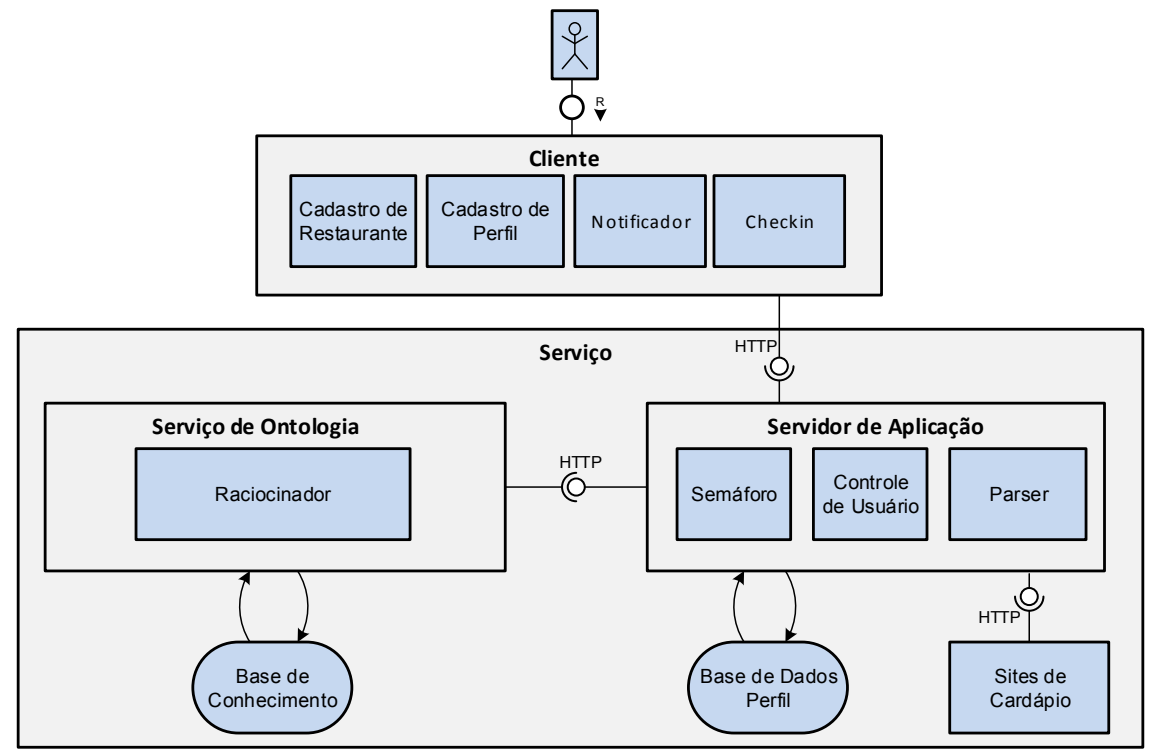

Figura 1. Arquitetura do Allergy Detector

O Servidor de Aplicação é constituído por três módulos principais: (i) Controle de Usuário: consulta na base de dados de perfil as informações de alergias do usuário a partir das credenciais do usuário informadas pelo módulo Cadastro de Perfil; (ii) Semáforo: inferir se o local é seguro, incerto ou inseguro e enviar informações para o módulo Notificador; (iii) Parser: obter dados de pratos/ingredientes do restaurante referente à URL informada pelo módulo "Cadastrar Restaurante".

Na Figura 2 é apresentado um diagrama com os três níveis do modelo que configuram a utilização de ciência de situação. A figura ilustra nove passos que representam a execução sequencial do processo de detecção de riscos de alergia baseado em ciência de situação, a saber:

1. Contexto de Localização: o módulo Checkin coleta as informações de localização através da internet (serviços de localização de IPs), triangulação de torres de telefonia celular (GPRS a partir do GSM) ou do GPS (rede de 24 satélites mundiais a uma altitude de $20200 \mathrm{~km}$ na faixa de frequencia UHF);

2. Contexto de Localização - Identificação Restaurante: O módulo Checkin identifica o nome do restaurante através de consulta a API do Google Maps utilizando a localização detectada;

3. Contexto de Localização/Perfil - Envio Informações Servidor de Aplicação: O módulo Checkin envia os dados de autenticação do usuário e o nome do restaurante para o servidor de aplicação;

4. Contexto de Perfil - Consulta Alergias: A partir da validação das credenciais do usuário, o módulo consulta na base de dados de perfil as alergias dele; 
5. Contexto de Perfil - Retorna Alergias: A base de dados retorna as informações de alergias do usuário para o servidor de aplicação;

6. Contextos Perfil/localização e Base de Conhecimento - Envio de Informações Serviço de Ontologia: O módulo informa ao serviço de ontologia o nome do restaurante e a lista de alergias do usuário;

7. Correlação de Contextos - Inferência Pratos com Alergênicos ao Usuário: O serviço de ontologia consulta a base de conhecimento em busca dos pratos servidos pelo restaurante que contenham ingredientes que sejam alergênicos ao usuário, inferindo seus derivados e as proteínas associadas aos ingredientes e aos pratos do restaurante;

8. Inferência - Envio de Informações Servidor de Aplicação: O serviço de ontologia informa ao servidor de aplicação as informações inferidas;

9. Inferência - Classificação Risco Local: caso o servidor de aplicação detecte que foram retornadas informações de pratos, ingredientes e URL, determinará a situação que o restaurante é um "Local Inseguro" e apresentará para o usuário quais pratos contêm ingredientes alergênicos ao usuário e suas proteínas. Caso o passo 8 não retorne nenhum valor, determinará a situação "Local Incerto" e caso retorne apenas a URL, indicará a situação "Local Seguro".

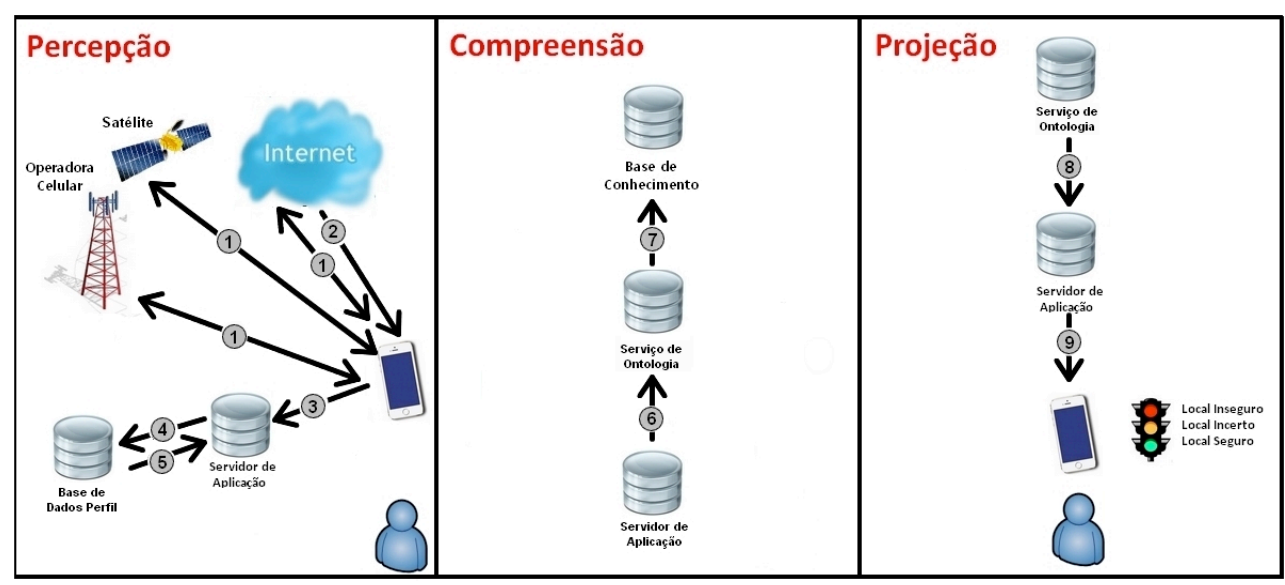

Figura 2. Diagrama de Ciência de Situação Baseado no Modelo de Endsley

Para permitir as inferências necessárias foi criada uma ontologia no domínio de alergia alimentar conforme ilustrado na Figura 3, contendo as classes Restaurante, Pratos_Restaurante, Ingredientes, Proteínas e as subclasses Oito_Principais_Alergênicos e Derivados_Dos_Oito_Principais_Alergênicos. A: Subclasse da classe Ingredientes que representa os derivados dos oito principais alergênicos.

O modelo foi projetado utilizando tecnologias abertas e gratuítas. No lado cliente foi gerado um aplicativo híbrido desenvolvido no framework Ionic, utilizando como linguagem de programação JavaScript, linguagem de marcação HTML e linguagem de estilos CSS, para smartphones compatíveis com Android e iOS. No lado Serviço desenvolveu-se o Servidor de Aplicação com linguagem de programação JavaScript sendo executado no NodeJS, a Base de Dados Perfil foi armazenada no banco de dados NoSQL mongoDB, o Serviço de Ontologia utilizou o framework Jena 
que utiliza linguagem de programação Java e que permite a linguagem de consuta SPARQL e a Base de Conhecimento foi gerada em OWL. O Servidor de Aplicação está armazenado na Plataforma OpenShift em um nó 1 small com 512MB de memória RAM e 1GB de storage, o Serviço de Ontologia e Base de Conhecimento estão armazenados na Plataforma Amazon Web Service (AWS) em uma instância t2.micro com 1GB de memória RAM e 30 GB de storage EBS e o mongoDB foi hospedado no MongoLab.

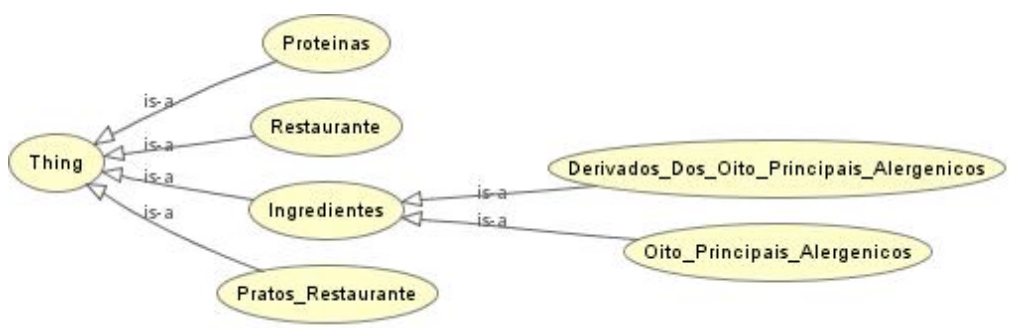

Figura 3. Ontologia Allergy Detector

\section{Avaliação e Discussão}

A comunidade científica tem utilizado cenários para avaliar projetos de computação móvel, ubíqua e ciente de contexto (Satyanarayanan, 2010; Zaupa et. al., 2012). Para avaliar o modelo proposto, foi desenvolvido um protótipo que realiza ciência de situação conforme proposto pelo modelo Allergy Detector. A ontologia foi modelada no Protègè $^{2}$ e exportada para Base de Dados de Ontologia no formato OWL ${ }^{3}$. Também foi utilizada a linguagem Java e biblioteca Jena ${ }^{4}$ para interação com a ontologia. Utilizou-se o seguinte cenário para avaliação do modelo:

"David Dunn é alérgico à amendoim, devendo evitar contato com a fonte alergênica. Ele possui o aplicativo Allergy Detector instalado em seu smartphone que fornece suporte na verificação do local onde deseja realizar refeição. Em um determinado dia, David Dunn está no centro de Porto Alegre quando decide fazer um lanche no Subway da Rua do Andradas próximo à Praça da Alfandega. Então David Dunn aciona a opção de Checkin do aplicativo Allergy Detector, para que seja verificado se são vendidos lanches que contenham amendoim como ingrediente. $O$ Allergy Detector apresenta um semáforo com uma luz vermelha na tela do smartphone do David Dunn, para informar que é um 'Local Inseguro', e mostra o nome do lanche 'Peito de Frango com Molho Teriyaki', que possui amendoim como ingrediente, mostrando ainda as proteínas do amendoim que são Ara_h_1, Ara_h_10, Ara_h_11, Ara_h_12 e Ara_h_13 (Figura 4a). Então David Dunn resolve ir no McDonalds próximo do Subway, onde aciona novamente o Checkin. Dessa vez o Allergy Detector apresenta um semáforo com uma luz verde e mostra a frase 'Esse restaurante não serve pratos que contenham amendoim' na tela do smartphone do David Dunn, para informar que é um 'Local Seguro' (Figura 4b)."

O Allergy Detector foi projetado para detectar riscos a usuários como David Dunn, que sofrem de alergia alimentar a um dos oito alergênicos mais comuns. $\mathrm{O}$

\footnotetext{
${ }^{2} \mathrm{http}: / /$ webprotege.stanford.edu;

${ }^{3} \mathrm{http}: / / \mathrm{w} 3$ org/TR/owl-guide;

${ }^{4}$ https://jena.apache.org;
} 
aplicativo exige o mínimo de intervenção do usuário, bastando que o mesmo mantenha atualizadas as suas informações de alergias, execute checkin no restaurante em que se encontre e, caso esse restaurante não tenha sido visitado por nenhum outro usuário ainda, é necessário informar o site onde são listados os pratos e ingredientes desse restaurante. $\mathrm{O}$ aplicativo alerta o usuário sobre a classificação de risco do local e informa ao usuário os nomes dos pratos contendo ingredientes alergênicos à sua saúde ou derivados desses ingredientes e as proteínas associadas aos mesmos. Devido aos restaurantes não disponibilizarem seus menus com a lista de ingredientes, definiu-se que o aplicativo contemplará os sanduíches de três grandes redes de fast-food que são o Habibs, McDonalds e Subway. O aplicativo também exclui os molhos customizados da Rede Subway, pois seria muito complexo ou impossível que a ontologia conseguisse inferir com precisão os ingredientes desses molhos.

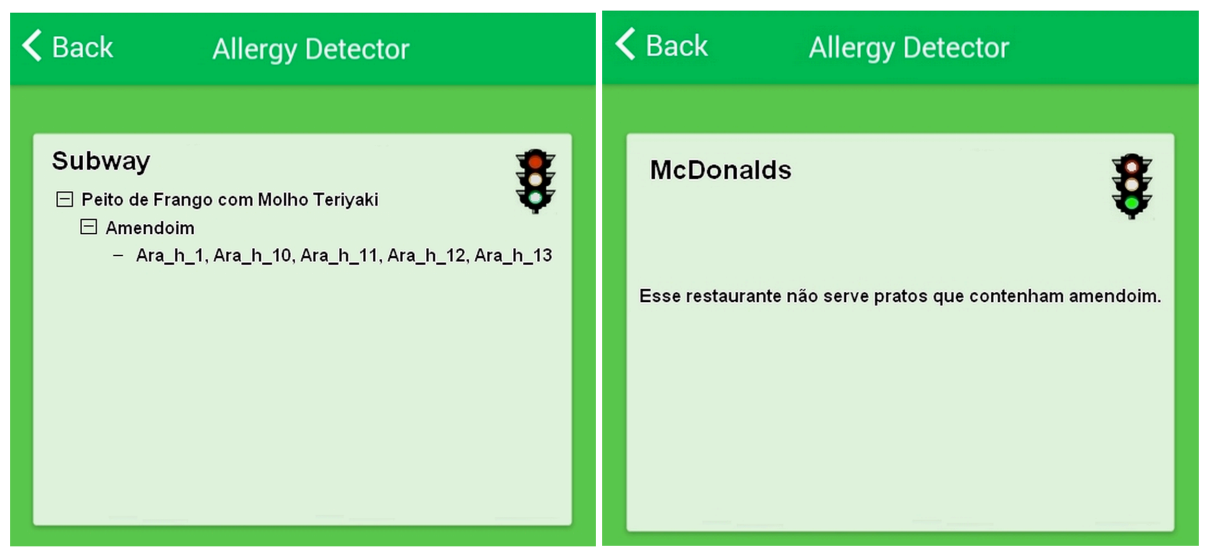

Figura 4. Telas do protótipo desenvolvido: (a) Local Inseguro (b) Local Seguro

Após a autenticação do usuário, o Allergy Detector aguardará que seja executada a função de checkin. Ao efetuar o checkin no restaurante, no qual David Dunn se encontra, o sistema aciona a API de Geolocalização do Google Maps, obtendo as informações de localização. Com essas informações o sistema descobre o nome do restaurante através da API do Google Maps. Nesse momento o Servidor de Aplicação recebe as informações de autenticação e do nome do restaurante. O Servidor de Aplicação por sua vez faz uma consulta com o nome do restaurante na Base de Conhecimento, para descobrir quais pratos e ingredientes o restaurante serve. Depois, faz outra consulta na Base de Dados de Perfil com as credenciais do usuário para descobrir os ingredientes que causam alergia ao usuário.

A partir das informações de alergias do usuário, dos pratos e ingredientes servidos no restaurante, o sistema infere se existe ou não a presença dos oito principais ingredientes alergênicos, ou de seus derivados, que causem alergia ao usuário, e quais são as proteínas associadas a esses ingredientes. Para inferir isso, o Serviço de Ontologia executa uma consulta SPARQL ${ }^{5}$ envolvendo o nome do prato, os ingredientes relacionados e as proteínas contidas nos pratos. Essa consulta é mostrada na Figura 5.

\footnotetext{
${ }^{5}$ http://www.w3.org/TR/rdf-sparql-query/
} 
A consulta SPARQL utiliza uma cláusula "SELECT" informando todas as variáveis de interesse, incluindo "?nomePrato", "?nomeIngrediente', "?nomeFonteAlergênica" e "?nomeProteína", uma cláusula "WHERE" com várias triplas como object properties "ContidoNoPrato", "ServePrato", "DerivaDe" e "ProteinaContida", bem como um filtro para trazer apenas informações das fontes alergênicas do usuário. Como resultado dessa consulta, são retornados todos os pratos servidos no restaurante em questão que possuem alergênicos ao usuário e as proteínas correspondentes dos ingredientes retirados da base de dados do IUIS/WHO.

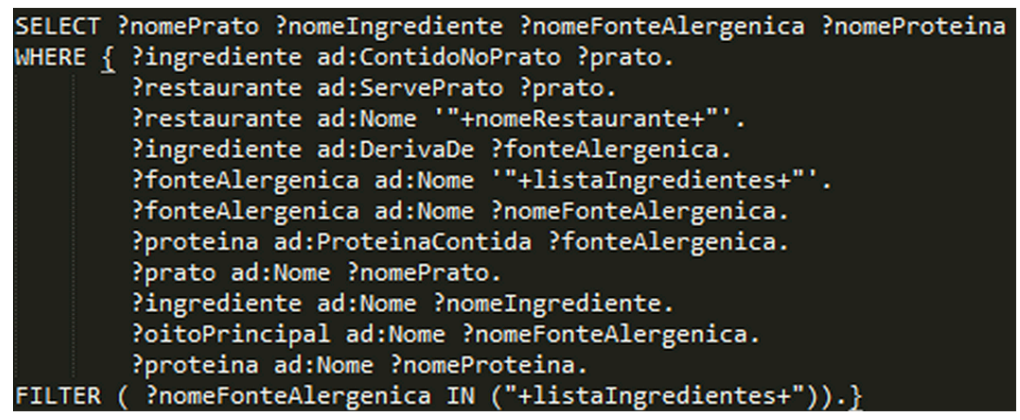

Figura 5. Consulta de Alergênicos ao Usuário Contidos nos Pratos

$\mathrm{Na}$ última etapa o servidor de aplicação busca no serviço de ontologia se foram encontrados pratos que possuem ingredientes alergênicos ao usuário, e em caso afirmativo inferirá que o restaurante em que o usuário se encontra é um "Local Inseguro". Caso contrário o servidor de aplicação deverá solicitar ao serviço de ontologia que faça uma consulta SPARQL na base de conhecimento para verificar se o restaurante possui Web Site com informações de pratos e ingredientes, e constatando que não possui Web Site, inferirá que o restaurante é um "Local Incerto" e se constatar que possui Web Site, inferirá que o restaurante é um "Local Seguro". Finalmente, o servidor de aplicação apresenta um semáforo representando as três classificações do local quanto ao risco de alergia alimentar (verde - seguro; amarelo - incerto; vermelho - inseguro). Por fim, apresenta também para o usuário, caso existam, os pratos que contém ingredientes alergênicos ao usuário e com as proteínas associadas aos mesmos, conforme mostrado na Figura 4.

O protótipo confirmou a expectativa de que a aplicação de ciência de situação, baseada no modelo de Endsley, possibilitaria que o modelo de forma ubíqua detectasse riscos ao usuário da presença de alergênicos nos pratos servidos nos restaurantes.

\section{Trabalhos Relacionados}

Foram pesquisados modelos relacionados com a área de saúde, tendo como enfoque alergia alimentar. Shoku-ping3 (Iizuka e Okawada, 2012) é um modelo que propõe apoio à seleção de menu de alimentação permitindo uma dieta diária segura levando em consideração restrições alimentares. São contemplados usuários que estão tomando remédios, sofrem de alergia alimentar e pessoas fazendo diálise. O modelo utiliza contexto de perfil dos usuários, tem interatividade com Base de Dados de Integração Alimento-Remédio e Composição Alimentar", acessado Web/dispositivos móveis.

PMR (Daraghmi e Yuan, 2013), Sistema de Restaurante Móvel Personalizado, propõe um modelo que dá suporte na escolha de restaurante a consumidores com diferentes perfis (religiões, culturas, alergias alimentares, condições de saúde, doenças 
crônicas, dieta especial, preferências e aversões). O modelo tem contexto de localização/perfil/nutricional, fornece recomendação(restaurantes/pratos/ingredientes) e possui interações Web/dispositivos móveis, pedidos de refeições e configuração remota.

Diário de Alimentação (Henricksen e Viller, 2012) propõe um modelo projetado para dar apoio a familiares no atendimento de crianças com reações alimentares. $\mathrm{O}$ modelo apresenta interações com os usuários, servindo como repositório dos alimentos ingeridos e trocas de informações entre usuários, como dicas de restaurantes e receitas.

Food Tracker (Johnson et al., 2014) é um modelo que disponibiliza acesso a usuários através de dispositivos móveis/Web a menus de restaurantes, informações nutricionais de itens de alimentação e dicas para alimentação saudável. O modelo apresenta contextos de localização/nutricional e recomendação de restaurantes.

A partir dos estudos relacionados, pode-se observar que os trabalhos utilizam algum tipo de contexto (localização ou de perfil). Porém nenhum deles utilizou outras características de computação ubíqua em seus modelos ou a combinação de diferentes contextos. Assim, foram identificadas como lacunas a utilização de ciência de situação e o uso de ontologias, abordagens empregadas no cerne do modelo Allergy Detector.

\section{Conclusão}

O modelo Allergy Detector permite detectar riscos de ingerir alimentos que desencadeiem reações alérgicas, através do uso de dispositivos móveis e da ciência de situação, em usuários que sofrem de alergia alimentar aos oito ingredientes alergênicos (soja, ovo, leite, trigo, peixe, crustáceo, amêndoas oriundas de árvores e amendoim). Ele emprega como contextos, para definição de situação, base de dados de alergia, informações de perfil, localização, e cardápios de restaurantes (pratos/ingredientes). Emprega também inferências em uma ontologia, o que possibilita a detecção de situações em que existam elementos alergênicos aos usuários, também agindo como um facilitador para ampliação da base de situações de risco. Tem como principal contribuição científica mostrar como uma aplicação de computação ubíqua aplicada à área de alergia alimentar pode funcionar com base na ciência de situação. Além disso, o modelo apresenta também uma importante contribuição para a sociedade, na detecção de riscos a usuários que sofrem de alergia aos oito ingredientes alergênicos.

Nesse estágio do trabalho, foi possível fazer a avaliação do modelo através da criação de um protótipo e emprego em um cenário, que demonstrou que a aplicação de ciência de situação possibilita alertar o usuário sobre o risco do local em servir pratos alergênicos ao mesmo. No primeiro nível do modelo, através do contexto de localização, foi possível obter as informações do nome do restaurante/pratos servidos e os ingredientes desses pratos. No segundo nível o modelo contextualizou as informações dos pratos/ ingredientes quanto à presença de um dos oitos principais alergênicos. Em seguida, fez uma correlação entre essas novas informações contextualizadas e as informações de contexto de perfil do usuário. No último nível o modelo conseguiu inferir, através da correlação de contextos do nível 2 , se o local traz riscos ou não ao usuário.

Esse modelo possui algumas oportunidades de trabalhos futuros, como a disponibilização das informações de alergênicos do IUIS em formato estruturado (XML) e a utilização de dados ligados para o registro do nome do restaurante, pratos e 
ingredientes, através dos componentes vocabulário (schema.org, GoodRelations, FOAF) e sintaxe (microdados, RDF-a, JSON-LD), que já se observam como padrões de ampla adoção. Nesse artigo descrevemos apenas o caso de uso, porém quando terminarmos a implementação, vamos avaliar a usabilidade e o desempenho, podendo surgir novas demandas para serem exploradas em trabalhos futuros.

\section{Agradecimentos}

Os autores gostariam de agradecer a CAPES, ao CNPq e a FAPERGS pelo apoio a essa pesquisa.

\section{Referências}

Antoniou, I. and Nanou, T. (2003) "An intelligent system for the provision of personalized dietary plans and health monitoring.", In: Information Technology Applications in Biomedicine, 2003. 4th IEEE EMBS Special Topic Conf., p. 70-73.

Arruda, L. K., Moreno, A. S. and Ferreira, F. (2013) "Molecular diagnosis of allergy: ready for clinical practice", In: Brazilian Journal of Allergy and Immunology (BJAI), 1(4), 187-194. doi:10.5935/2318-5015.20130024

Daraghmi, E. and Yuan, S. (2013) "PMR: Personalized Mobile Restaurant System.", In: Computer Science and Information Tecnology (CSIT), p. 275-282.

Endsley, M. (1999) "Situation awareness in aviation systems.", In: Handbook of Aviation Human Factors, 257-276.

Feltes, L. H. and Barbosa, J. L. V. (2014) "A Model for Ubiquitous Transport Systems Support.”, In: Revista IEEE America Latina, v. 12, n. 6, p. 1106-1112.

Gelogo, Y. and Kim, H. (2013) "Unified Ubiquitous Healthcare System Architecture with Collaborative Model.”, In: International Journal of Multimedia and Ubiquitous Engineering, v. 8, n. 3, p. 239-244.

Guandalini, S., \& Newland, C. (2011) "Differentiating food allergies from food intolerances." In: Current Gastroenterology Reports, 13(5), 426-34.

Henricksen, K.and Viller, S. (2012) "Design of software to support families with foodallergic and food-intolerant children.”, In: Proceedings of the 24th Australian Computer- Human Interaction Conference, p. 194-203.

Iizuka, K. and Okawada, T. (2012) "Food menu selection support system: considering constraint conditions for safe dietary life.", In: Proceedings of the ACM multimedia 2012 workshop on Multimedia for cooking and eating activities, p. 43-48.

Johnson, T., Vergara, J., and Doll, C. (2014) "A Mobile Food Recommendation System Based on The Traffic Light Diet", p. 1-8, 2014. Disponível em: http://www.arxiv.org/pdf/1409.0296. Acesso em 10/10/2014

Pereira, A. da S.; Moura, S.; Constant, P. (2008) "Alergia alimentar: sistema imunológico e principais alimentos envolvidos Food allergy", In: system immunologic and main food involved. uel.br, p. 189-200.

Satyanarayanan, M. (2001) "Pervasive computing: Vision and challenges", In: Personal Communications, IEEE, 8: $10-17$.

Silva, L. R. et al. (2008) "Consenso Brasileiro sobre Alergia Alimentar: 2007" Documento conjunto elaborado pela Sociedade Brasileira de Pediatria e Associação Brasileira de Alergia e Imunopatologia, 64-89.

Zaupa, D.; Costa, C. and Silva, J. (2012) "Implementing a spontaneous social network for managing ubiquitous interactions.”, In: Computer Systems (WSCAD-SSC), 2012 13th Symposium on, p. 163-170. 